\title{
First Powering of the LHC Test String 2
}

\author{
Frédérick Bordry, Davide Bozzini, Knud Dahlerup-Petersen, Reiner Denz, Bruno Puccio, Adriaan Rijllart, \\ Felix Rodriguez-Mateos, Roberto Saban, Rüdiger Schmidt, Luigi Serio, and Hugues Thiesen
}

\begin{abstract}
String 2 is a full-size model of a regular cell in an LHC arc. In the first phase, three dipole magnets and two quadrupole magnets have been assembled in String 2 and commissioning started in April 2001. By the beginning of 2002 three pre-series dipole magnets will be added to complete the cell.

As for its predecessor String 1, the facility was built to individually validate the LHC systems and to investigate their collective behavior for normal operation with the magnets at a temperature of $1.9 \mathrm{~K}$, during transients as well as during exceptional conditions.

String 2 is a precious milestone before installation and commissioning of the first LHC sector (1/8 of the machine) in 2004, with respect to infrastructure, installation, tooling and assembly procedures, testing and commissioning of individual systems, as well as the global commissioning of the technical systems.

This paper describes the commissioning, and retraces the first powering history.
\end{abstract}

\section{INTRODUCTION}

$\mathbf{S}$ TRING 2 is being assembled in two phases [1]-[3]. The facility, which was commissioned during the first phase, includes one half-cell with three $15-\mathrm{m}$ long dipoles and one short straight section (SSS) which in turn is an assembly of a quadrupole magnet and several correctors. The SSS of the second half-cell was also installed. For Phase 2, three pre-series dipole magnets will be added early in 2002 . The prototype magnets are full-scale magnets similar to the series magnets but heavily instrumented for the experimental program. String 2 has the same set of corrector magnets as for an LHC cell.

The electrical feed-box (DFBS) [4] is installed at the upstream end. It is a prototype of the arc feed boxes and contains the high temperature superconducting (HTS) current leads [5] through which the 11 of the 15 electrical circuits are fed. The magnet return box is installed after the last SSS on the downstream end. It contains the short circuits and the jumper connection simulating the connection to the cryogenic distribution line (QRL) of the following cell. The QRL runs parallel to the magnets for the distribution and recovery of helium at various temperatures and pressures [6].

String 2 has a length of about $120 \mathrm{~m}$ and follows the curvature as the machine in the LHC tunnel. Because it comprises the cryogenic line, an artificial difference in level was required to simulate the trench in the tunnel. This was achieved using prefabricated concrete slabs.

With respect to vacuum, cryogenics, interlocks, protection and powering, Phase 2 of String 2 represents a full cell in the regular part of the LHC arc and allows therefore a partial validation of the several major LHC subsystem.

Manuscript received September 28, 2001

The authors are with CERN, Geneva, Switzerland.

Publisher Item Identifier S 1051-8223(02)03465-6.

\section{THE ElECTRICAL CiRCUITS}

The dipole circuit [7] includes an energy extraction system and can be configured to include one or two power converters in series. A thyristor converter is used to ramp the current up or down while a switch-mode converter is used during injection and the flat top phases. It also acts as an active filter during ramping.

The multipole correctors are small single aperture magnets placed at either ends of each dipole inside the cold masses to compensate for the field errors generated by the dipoles. All dipole cold masses contain one sextupole corrector for each aperture. The sextupoles for each beam are powered separately. Every second dipole cold mass contains one decapole corrector for each aperture. As for the sextupoles, the correctors for each beam are powered separately.

There is one circuit with focusing quadrupoles and one with defocusing quadrupoles in String 2. Each SSS contains one of each: each beam sees alternately one focusing quadrupole and one defocusing quadrupole. There is no energy extraction system for the quadrupole circuits.

The sextupole and octupole lattice correctors in the SSS are powered via the superconducting cable running in a superfluid helium-filled tube attached to the magnet cold masses. Each corrector type is powered in two such circuits. For the sextupole lattice corrector circuits an energy extraction system is installed.

The orbit corrector dipole magnets in the SSS are separately powered using local $60 \mathrm{~A}$ power converters. These are the only circuits, which are not fed via the DFBS.

\section{QUENCH DETECTION}

Floating bridge detectors are used to detect a quench in any of the main magnets. Every detector has three identical channels evaluating the resistive voltage increase. A quench is validated when at least two out of the three channels within the same detector exceed the threshold $(110 \mathrm{mV})$ for a time longer than a pre-determined period $(10 \mathrm{~ms})$. Quenches in the interconnecting bus-bars of a circuit are detected with special differential voltage detectors. The inductive component of the measured voltage across the circuit is subtracted using measurements of the voltage drop across individual magnets. A comparison of the remaining (resistive) part with a pre-defined threshold gives a quench signal with the two out of the three mechanism described above.

For corrector circuits, quenches are detected by means of differential detectors, which eliminate the inductive component of the measured voltage signal (global quench detectors). The current in each circuit is measured, and by appropriate processing, the inductive voltage across the magnets is calculated. 
This value is then compared with the total measured voltage to detect any resistive voltage generated by a quench in the circuit (magnet or bus-bar).

Particular attention has been given to the protection of the current leads. The upper part of a lead is made with copper and the lower part is made with HTS material. Differential detectors monitor the voltage drop across the superconducting part of the lead. In order to provide a redundant detection, temperature sensors are included in the quench detection. These sensors are mounted at the interface between the resistive and superconducting part. The voltage drop across the resistive part is monitored and compared to threshold values.

The Quench Protection System in String 2 includes an independent, distributed acquisition and monitoring facility as the one to be installed in the LHC tunnel and the underground areas. Dedicated processors send information concerning status and other variables of the protection equipment to a supervision application. They also provide information about transients through local acquisition buffers where the signals are recorded in case of quench or other events.

About 400 signals are recorded: they include voltages, temperatures, and signals from Hall probes. The setting of the gain factors in the amplifiers is done with a computer, accessing the local signal conditioners via a field-bus. The values of the gains are also transmitted to the general-purpose data acquisition system and are used for calibration.

\section{Power Permit And Power Abort}

To permit powering of the magnets, several conditions need to be satisfied (Power Permit). The temperature of the magnets must be below $2 \mathrm{~K}$, the capacitors for the quench heaters must be charged and the energy extraction system must be ready. The data acquisition system is armed to record post mortem data in case of a quench or another failure. In case of a quench, a fast stop (Fast Abort) of the power converter is issued. In those circuits where it is present, the switch for energy extraction is opened. For other types of failures, such as a slow drift of the magnet temperature exceeding $2 \mathrm{~K}$, the power converter is slowly ramped down (Slow Abort). The interlock system uses a combination of an industrial PLC and a hardware matrix [8].

\section{COMMISSIONING OF THE CRYOGENIC SYSTEM}

The cryogenic system of String 2 comprises the cooling loops for the string of magnets, the cryogenic distribution line, the electrical feed box, the feed and return boxes, the transfer line and the refrigerator [9], [10]. The latter four were tested at operating conditions prior to the installation of the magnets.

After the string assembly and in parallel with the pressure and leak testing, the components and instrumentation of the cryogenic system were checked at ambient temperature to verify their integrity and correct wiring and connection.

With the cool down, the instrumentation was tested and the control loops tuned while they were put in service.

The refrigerator was tuned to supply the necessary cooling power at various temperatures within the constraint of $75 \mathrm{~K}$ maximum temperature difference across each magnet. The tur-

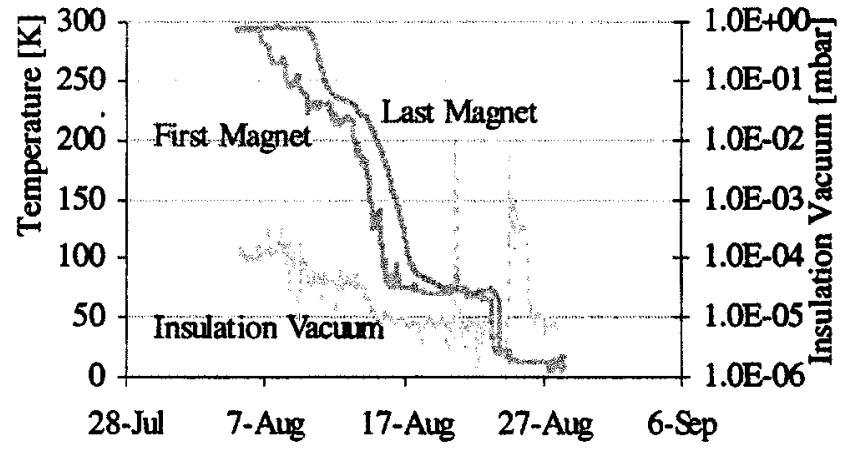

Fig. 1. The cool down.

bines and the liquid nitrogen pre-cooler were put into operation progressively and the high pressure feeding increased.

The refrigerator, in series with the magnet string and the electrical feed box, progressively cooled down the transfer lines, the lines feeding the QRL and the thermal screens. Once below $100 \mathrm{~K}$, the fine-tuning of the thermal screen loops was performed.

After liquid helium temperature was reached, all the liquid helium gauges were switched on and their respective control loops activated and tuned. While the magnets were filled with liquid helium, the cryogenic system was ready to be coupled to the pumping group for the final cool down to $1.9 \mathrm{~K}$. At nominal operating conditions all control loops were tuned, the instrumentation verified and the temperature levels and heat loads assessed. Further tuning was performed on the superfluid helium loop, on the liquid helium level in the DFBS and the cooling circuits for the current leads during progressive powering.

The process control, alarms, interlocks and signals exchange with other systems were verified and adapted to the operating conditions encountered during and after cool down.

Apart from the unavoidable time spent for troubleshooting on components and instrumentation, the overall cool down of the magnet string from $300 \mathrm{~K}$ down to $1.9 \mathrm{~K}$ took about 2 weeks (see Fig. 1).

\section{COMMISSIONING OF THE ElECTRICAL Systems}

The electrical systems were commissioned in two phases. The first phase is a separate commissioning of the elements of the warm part of the electrical circuits (power converters, water cooled DC cables and energy extraction systems) and those of the cold part (the string of magnets, the current leads, the quench detectors and quench heaters). In the second phase, all elements are put together by connecting the warm DC cables to the current leads.

The first phase included the verification of the integrity of the circuits, in particular magnet coils and instrumentation. The performance of the power converters was validated in conjunction with the warm DC cables and water-cooling system. The correct functioning of the interlock system was verified by simulating various failure conditions.

The second phase started with the gradual powering of the electrical circuits from minimum stable current, through injection current up to nominal current. 
TABLE I

EleCtrical InSUlation TEST OF THE DipOle CiRCUIT

\begin{tabular}{cccc}
\hline Temperature [K] & Voltage $[\mathrm{V}]$ & Duration $[\mathrm{s}]$ & Leakage $[\mu \mathrm{A}]$ \\
\hline 300 & 200 & 120 & 0.76 \\
90 & 200 & 120 & 1.42 \\
1.9 & 500 & 120 & 8.1 \\
\hline
\end{tabular}

The commissioning of the electrical systems was not only well defined in advance, but also carefully documented as the tests were carried out.

\section{A. Power Converters}

All 15 converters were tested at full power (heat run test, performance measurement). The converters of the dipole circuits were tested on a water-cooled dummy load. The internal and external interlocks of the power converters were tested. Power Permit, Fast Abort and Slow Abort were individually tested for each converter in conjunction with the interlock system.

The high-precision digital current control loops set for resistive loads and ramping were successfully tested: no lagging current error and no overshoot [11] was detected. Voltage ripple measurements were performed and found to be compatible with the requirements for LHC.

\section{B. Electrical Tests of the Circuits One by One}

The electrical tests of the circuits aimed at qualifying the status of the circuits before, during and after the cool down. They comprise electrical insulation tests, measurements of the resistance of quench heater strips, the continuity test of all diagnostic instrumentation and the transfer function measurements of global and partial circuits. The integrity and the correct polarity of the quench protection diode installed in parallel to each magnet was verified. All these tests were done at warm and repeated at $90 \mathrm{~K}, 20 \mathrm{~K}$ and, depending on the test, also at $1.9 \mathrm{~K}$.

The electrical insulation of each single circuit was measured with respect to the other electrical circuits, to the quench heaters, to ground and to any other electrical instrument mechanically installed in the vicinity of the tested circuit. $200 \mathrm{~V}$ DC have been applied at warm and during the cool down, at nominal cryogenic conditions the voltage withstand was increased to $500 \mathrm{~V}$. The duration of the test was 2 minutes after which the leakage current was measured. Table I shows the main parameters and the results of the electrical insulation test of the dipole circuit at the different temperature levels.

The continuity of the diagnostic voltage taps was tested to verify the connection of each wire in its correct position on the circuit.

The resistance of the quench heaters was measured at warm and at $20 \mathrm{~K}$ to determine the residual resistivity ratio (RRR).

The transfer function of the magnets was measured at warm, $90 \mathrm{~K}$ and at $1.9 \mathrm{~K}$ to verify the integrity of the magnet coils and to provide the input for the calculation of transmission line effects.

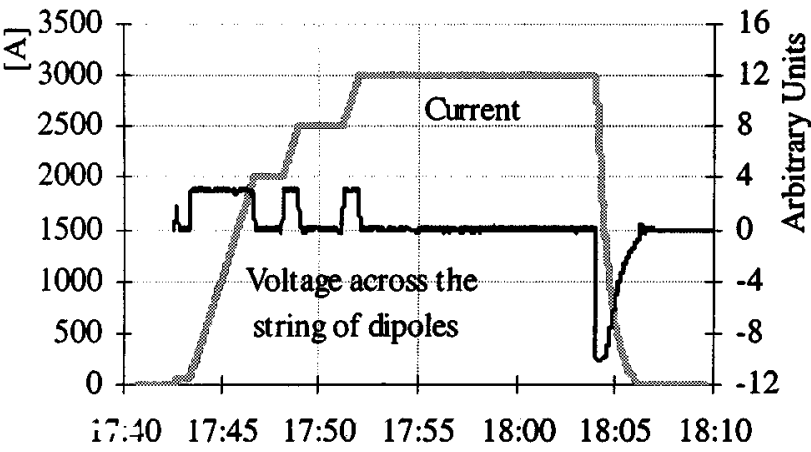

Fig. 2. Discharge from $3000 \mathrm{~A}$.

\section{Interlock Tests}

These tests took place with the magnets not connected to the power converters. The latter were run on short circuits or dummy loads. The test mimicked a full power test for the protection, the interlocks and the energy extraction systems. The power converters were powered and all the possible inputs to the interlock system pertaining to each circuit were activated. The outcome was recorded and verified that it matched the expectations. Interlock signals for the dipole circuit are quench, water failure in the cables, switch open failure, power converter failure.

The PLC used to authorize the powering of each circuit (Power Permit) was tested. Various combinations of input states for the PLC were set, and it was verified that the Power Permit for the converter was given only for the correct set of inputs states.

\section{Initial Commissioning of the Electrical Circuits at Low Current}

After the power converters were connected to the circuits, the tests described in Section VI-C were repeated. The current was set to the minimum stable value that can be handled by the power converter, $50 \mathrm{~A}$ for the dipole circuit and $100 \mathrm{~A}$ for the quadrupoles.

\section{E. Commissioning of the Electrical Circuits Up to Nominal Current}

Several tests were carried out at various current levels to verify the correct functioning of all equipment for protection of the magnets (quench detection, energy extraction, quench heaters and interlock system). The tests included the opening of the switch of the energy extraction system and the firing of the quench heaters plus subsequent detection of the quench. The tests carried out at a current of 200 and 800 A confirmed the correct functioning of the equipment.

Already during a discharge from a current of $800 \mathrm{~A}$, the global quench detectors on the dipole bus-bars between the DFBS and the first quadrupole were saturated.

The tests continued with a discharge and firing of heaters with subsequent quench detection at 2.0,3.0 and 6.5 kA.

An inversion of voltage taps across the resistive part of one of the HTS current leads was discovered and repaired. A faulty temperature sensor required for the protection of the lead was replaced with an adjacent sensor. 


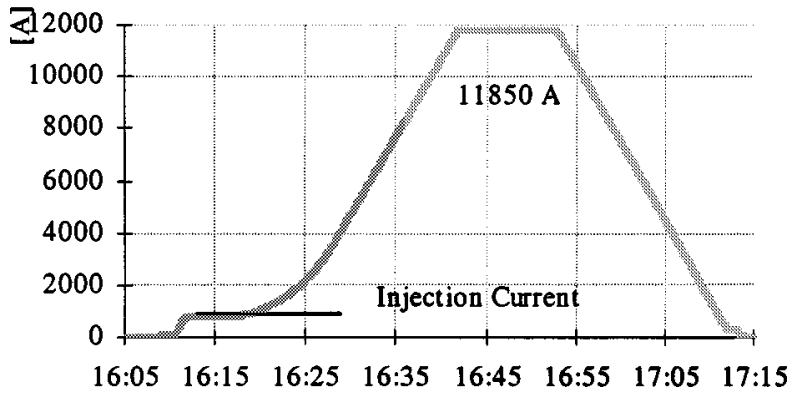

Fig. 3. Current cycle to nominal current, and back to zero.

In order to avoid the (mis-)firing of the global quench detectors on the dipole bus-bars; the signal from the inductive voltage across the dipoles during the current decay was subtracted. The threshold was raised to $200 \mathrm{mV}$ but during a discharge from $9 \mathrm{kA}$ the signal was well above this threshold $(550 \mathrm{mV})$ and the heaters were fired on two out of three dipoles. This test was aimed at verifying the performance of the dump switch at $9 \mathrm{kA}$.

\section{F. Commissioning of the Electrical Circuits Powered in Unison at Nominal Current}

When the other circuits are commissioned, it is planned to ramp the current in all circuits together to measure the tracking between these currents. Three fixed coils are installed inside the beam apertures, one for each quadrupole circuit and one for the dipole circuit. When the magnet field is ramped, the inductive voltage is measured and allows a measurement of the correct tracking at the level of the field. These fixed coils should allow the measurement of the transfer function between the magnet field and the current.

\section{FIRST POWERING}

At the time of writing, the three main circuits were commissioned and powered up to nominal current.

The ramp to nominal current of 11850 A for the dipole circuit [12] starts slowly and continues with a maximum ramp rate of $10 \mathrm{~A} / \mathrm{s}$ after several minutes. At the first attempt, the second dipole magnet quenched 230 A below nominal current. In the following ramp nominal current was reached without quench. Both circuits with quadrupole magnets reached nominal current without quench.

\section{CONCLUSIONS}

The String 2 systems involved in the powering and in the preparation for powering performed as expected. The methods used to verify the equipment highlighted faulty components or errors which were easily identified and corrected. In this first phase of operation, String 2 has fulfilled its objective by offering the means to verify the commissioning procedures, which will be refined and used for the commissioning of the LHC sectors one by one.

\section{ACKNOWLEDGMENT}

The authors would like to thank P. Proudlock for his contribution to the definition of the electrical commissioning, P. Gomes and S. Pelletier for painstakingly commissioning the cryogenic sensors and T. Ladzinski for the beautiful tool to configure the data acquisition system. Last but not least, we would like to thank all the teams in the LHC, EST, SL and ST Divisions of CERN who made String 2 a reality.

\section{REFERENCES}

[1] F. Bordry et al., "The LHC test string 2: Design study,", LHC Project Report, March 1998.

[2] R. Saban et al., "The preparation of the cryomagnets and the assembly of the LHC test string 2," in PAC'01, Chicago, June 2001.

[3] F. Bordry et al., "The LHC magnet string program: Status and future plans," in PAC'99, NY, Apr. 1999.

[4] $\mathrm{Ph}$. Sacre et al., "The electrical distribution feedbox for the LHC prototype cell," in EPAC'00, Vienna, Austria, June 2000.

[5] A. Ballarino, "Applications of high temperature superconductors to accelerators," in EPAC'00, Vienna, Austria, June 2000.

[6] W. Erdt, G. Riddone, and R. Trant, "The cryogenic distribution line for the LHC: Functional specification and conceptual design," in Adv. Cryo. Eng 45. New York: Plenum Press, 2000, pp. 1387-1394.

[7] R. Saban et al., "The electrical circuits of string 2,", LHC-XMS-ES0002, LHC Eng. Spec., May 2001.

[8] B. Puccio et al., "The interlock system for string 2: Power abort and power permit,", LHC-XMS-ES-0001 LHC Eng. Spec., Sept. 2000.

[9] L. Serio, "The cryogenic circuits of string 2,", LHC-XMS-ES-0003, LHC Engineering Specification, Oct. 2000.

[10] —, "The process of the cryogenic system for string 2: Functional analysis,”, LHC-XMS-ES-0004, LHC Eng. Spec., Feb. 2001.

[11] F. Bordry and P. Proudlock, "LHC powering from string 2 to sector test," in Proc. 11th Chamonix Workshop, Jan. 2001.

[12] P. Burla, Q. King, and J. G. Pett, "Optimization of the current ramp for the LHC," in PAC'99, New York, Apr. 1999. 